\title{
Flexible moving targets research based on contour coefficient variation analysis and video detection
}

\author{
Tianshu Zhang, Di Mou, Wenshi Ren and Zhenghua Feng \\ School of China Three Gorges University, Yichang 443000, China
}

\begin{abstract}
This paper through the existing video files, moving object is obtained by matlab programming background subtraction division of the foreground image, to extract the contour parameters. Contour parameters of the foreground image can be gained by analyzing the variation coefficient, then through the analysis of the variation coefficient can be used as a flexible moving targets in the process of video detection and rigid motion target identification features.
\end{abstract}

KEYWORD: Matlab; Contour extraction; Foreground image; Coefficient of variation

\section{INTRODUCTION}

Many applications of image processing technology have brought a lot of new ideas to the electric power industry, which have solved some problems that have not been solved before. Because of its high processing precision and rich content, it has many advantages, such as wide application, good reproducibility and good flexibility, which has been shown in the practical application. With the development of computer and related technology, image processing technology is becoming more and more important in the electric power industry of our country and the range of applications will be wider. Extraction and tracking of moving object is the key link of video surveillance. Video surveillance is the analysis and processing of the image sequences obtained by the camera, the main purpose is to observe the moving objects of the scene in real time, and to analyze their behavior. The research of this technology is mainly related to motion detection and object extraction, object classification, object tracking, object recognition behavior analysis and so on.1)Motion detection: video data from the shot through the background modeling, motion segmentation and other motion detection method to detect the movement of the region.2)Object extraction: after detected motion area, and determines whether the region's real target or background shaking, extracted real moving target. Target classification: the classification of moving targets, divided into two major categories of rigid and non-rigid, in order to facilitate the subsequent different tracking processing. Object of this study was rigid target. Research status: in recent years, scholars around the world for the video image moving target detection and tracking problem do large and in-depth research, put forward many effective methods, but also made some progress, but so far and does not appear to can be suitable for various occasions and conditions of the general algorithm. There are various algorithms that have kinds of flaws in the robustness, accuracy and reliability etc. Source of difficulty is mainly because of interference factors exist in the video. These factors include: target motion displacement or deformation of itself, the imaging sensor itself inherent noise, lighting changes, background clutter and moving target of self-occlusion or mutual occlusion and so on. These questions need to be further studied.

\section{USING BACKGROUND SUBTRACTION TO OBTAIN A FOREGROUND IMAGE.}

The effective extraction of the moving object contour is very important for the understanding of the target recognition, tracking and behavior. Influenced by the complexity of the background, when the background intensity is similar to the gray level of the moving object, the moving object is easy to generate holes, so some parts can not be fully recovered. In this paper, we use the background subtraction method to detect moving regions, and the difference images for dynamic threshold segmentation, as well as the edge of the link, and then make its edge in a basic continuous state. The obtained binary image to extract the contour and area threshold are selected according to the size of 
the target, excluding noise or background extraction caused by unclean the false contour. We will get the contour mask .Then use contour mask image obtained with the original image to make logic operation to extract aims. Background subtraction, also known as background subtraction, the principle of background subtraction is the moving image and the background image of the current frame differential motion to obtain the target area, this method can be more better than the frame difference method to identify and extract the moving target. This is a method most commonly used. However, background subtraction need to build a background image, the background image must be free piece of moving targets, and should be able to adapt to constantly update the current changes in the background. To get the background image $b(x, y, t)$, the simplest way is the first frame without moving object in the image frame as a background image, because no fireworks in the first frame of the image motion and changes in the background is very small, so here we can select the first frame of the image as the background image. Then use the background difference method, respectively, the 89th frame, 90th frame, the 91 frames frames fireworks moving foreground image (image shown in Table 1 in the picture).We selected sixteen sets of image data as a reference. Firstly the foreground image was subjected to a filter and a secondary filter, a secondary filter image obtained by binarizing process, select two groups of varying sizes can be programmed threshold value when binarization processing, and then to get the two groups image edge detection. The median filtering method is effective to remove salt and pepper noise, in the phase of the optical measurement of fringe image analysis processing method has a special function, but in the center of the stripe analysis method has little effect. Median filter in image processing, commonly used to preserve the edge information is classical noise smoothing method.

Median filter has a good filtering effect on the impulse noise, especially when the noise is filtered. It can protect the edge of the signal, so that it can not be blurred. These excellent properties are not the same as the linear filtering method. In addition, the median filtering algorithm is relatively simple, but also easy to use hardware to achieve. So, the median filtering method is put forward, then get the important applications in the digital signal. Median filtering method: When filter a digital signal sequence $x_{j} \mathrm{j}(-\infty<\mathrm{j}<\infty)$, we must first define the length of a long window, which length is L. $L=2 N+1, \mathrm{~N}$ is a positive integer. At a certain moment, the signal sample in the window is $x(i-N), \ldots, x(i), \ldots, x(i+N)$, Where a is located in the center of the window signal sample value. This signal sample values in ascending after a large order, which samples at L, it is defined as the median filter output values, written as shown in the median filter under the "minimum absolute error" criterion best excellent filtering. In practical applications, with the increase of the length of the selected window, the computation of the filter will increase rapidly. Therefore, it is an important research content of the theory of median filtering to seek the fast algorithm of median filter.

Median filter and edge detection: the use of the foreground image function reads the motion has gotten the fireworks, fireworks on the motion .The foreground image obtained by median filtering and edge detection of moving object (fireworks) edge image, median filtering can keep target edge, which is the median filter with respect to the mean filter greatest advantage. Median filtering denoising performance, eliminate isolated noise points can be used to weaken the random interference and pulse interference, but the edges are not blurred.

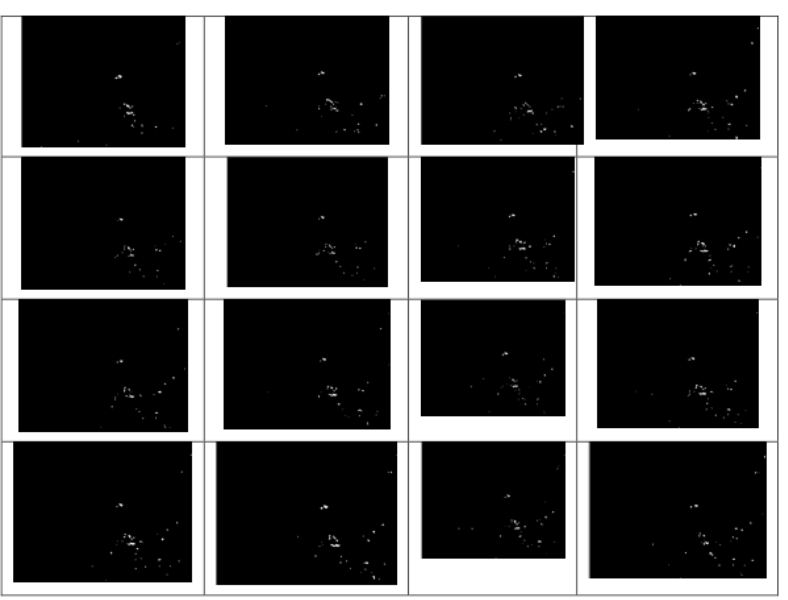

Fig. 1 Image table of edge detection

\section{USING MORPHOLOGICAL PROCESSING TO THE EDGE OF THE IMAGE}

Mathematical morphology is a subject based on rigorous mathematical theory, which has become the theoretical basis of many image processing and computer vision technology, and is widely used in the extraction of contour. Mathematical morphology is a nonlinear filtering method. Among them, the binary mathematical morphology transform is a collection process, basic operations including corrosion, swelling, open operation and close operation. The essence of these operations is expression objects or the shape of the set and structural element of interaction. The shape of the structure elements here will determine the shape information of the signal . he idea of morphological edge extraction is: after a certain transformation, the 
gray value of the image edge to be extracted is significantly more than the non edge part. A variety of methods have been proposed to extract the contour of the target, using morphological theory.

Using the same method to get the rest of the various groups of fireworks outline image (larger wide value), will get the outline of the image summary list which is shown in the following table.

\section{BY DETECTING THE EQUIVALENT} DIAMETER AND OTHER PARAMETERS TO ANALYZE THE VARIATION COEFFICIENT

Through the above series of analyzes and calculations, we have obtained a series of moving targets (fireworks) of the foreground image of the contour, and next, we will get the equivalent area and equivalent perimeter of the contour of the moving target (fireworks) of the foreground image, the equivalent diameter of the moving target is obtained. By analyzing the equivalent diameter of the moving target (fireworks), can distinguish the moving target (fireworks) is a flexible or rigid moving target.

Calculate the equivalent diameter and other parameters

Using imread function to read the edge image, using the Areas function to calculate the equivalent area of fireworks, using Perimeter to calculate the equivalent perimeter of fireworks: The calculation of equivalent area and equivalent length of fireworks, obtained by calculating the equivalent diameter and equivalent perimeter and then calculated the equivalent radius: $\mathrm{R} 1=\mathrm{S} 1 / \mathrm{L} 1=3.78, \mathrm{R} 1{ }^{\prime}=\mathrm{S} 1 / \mathrm{L} 1=$ 3.76 .

Similarly you can obtain data from other groups, we will be obtained from the 2 set of equivalent radius of the list, get the next table:

Table 1 Equivalent radius Table (A larger threshold value)

\begin{tabular}{llll}
\hline $\mathrm{R} 1=3.78$ & $\mathrm{R} 2=3.11$ & $\mathrm{R} 3=2.85$ & $\mathrm{R} 4=3.09$ \\
\hline $\mathrm{R} 5=3.33$ & $\mathrm{R} 6=2.90$ & $\mathrm{R} 7=3.29$ & $\mathrm{R} 8=2.57$ \\
$\mathrm{R} 9=2.31$ & $\mathrm{R} 10=3.08$ & $\mathrm{R} 11=2.97$ & $\mathrm{R} 12=3.13$ \\
$\mathrm{R} 13=2.98$ & R14=3.61 & R15=3.11 & R16=2.63 \\
\hline
\end{tabular}

Table 2 Equivalent radius Table (A smaller threshold value)

\begin{tabular}{llll}
\hline R1=3.76 & R2=3.54 & R3=3.72 & R4=3.16 \\
\hline R5=3.31 & R6=3.45 & R7=3.76 & R8=3.15 \\
R9=2.60 & R10=3.38 & R11=2.97 & R12=3.51 \\
R13=3.09 & R14=3.62 & R15=3.06 & R16=3.30 \\
\hline
\end{tabular}

\section{IDENTIFICATION OF FLEXIBLE MOVING} TARGET AND RIGID MOVING TARGET

According to the tracking object, the moving object can be divided into the rigid moving object and the non rigid moving target, rigid target refers to the external force, the shape and size of the object (size) remain unchanged, and the relative position of the various parts of the interior remains constant (no distortion) is the ideal physical model, the non rigid moving target is a flexible target. The deformation of the target is involved in the deformation of the target, such as the rotation and the shape of the target. At present, the research on non rigid moving target is divided into two major categories, the first class is based on the contour of the target tracking. One is to track the target's color to track the target when the target is deformed. In this article we take the contour moving target research. By analyzing the movement of the target compares the calculated (Fireworks) equivalent diameter can be learned, The change rate of the equivalent diameter is not the same, that is the shape and size of the object has changed, and the outline of the moving target (fireworks) has changed, through the above analysis we can know that the movement of fireworks is a flexible movement. By detecting and analyzing research on flexible moving target (Fireworks), can detect the security of transmission line operation, and this is very important for the research and detection of power system (Figure 2).

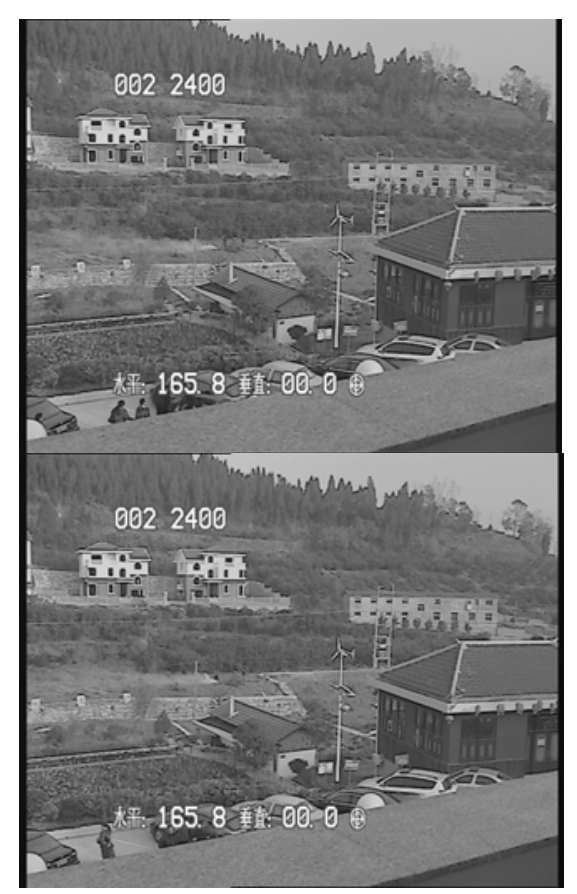

Fig. 2 comparative analysis

From figure 2 we can get that, the shape and size of the car remains the same, it can be extracted feature region having a relatively stable area; the relative positions of the various parts of the car interior remains constant, the actual edge large distortion does not occur within a certain segment of the image sequence; vehicle movement state has a certain continuity. So the car movement is a rigid movement. And the shape and size of the fireworks 
has changed, the contour is slowly changing, so the movement of fireworks is flexible. In the above picture, we can take trees shaking in the picture as a noise. In the outline of the moving target coefficient of variation analysis process, in this paper, the method of equivalent radius is used. The binary image segmentation of moving targets to achieve. Binary image was subjected to area calculation, that is, the number of targets at this time; then extract the target image side, edge extraction by using the segmentation image, get side length [6].The equivalent radius is calculated by using the formula (1).

$$
\rho_{\mathrm{b}}=\frac{\mathrm{L}_{b}^{2}}{4 \pi A_{b}}
$$

To get moving object silhouette coefficient of variation of the complexity of the analysis, in this paper, we use the Hurst index to analyze the random properties of the time series of the moving target contour. Hurst proposed a new statistic, That Hurst exponent, and proposed rescaled range $\mathrm{R} / \mathrm{S}$ analysis method.

$$
\begin{aligned}
& (R / S)_{n}=A n^{H} \\
& R=\max \left(X_{t, n}-\min X_{t, n}\right)
\end{aligned}
$$

In the formula: $\mathrm{n}$ is the number of samples, $\mathrm{Xt}, \mathrm{n}$ represents the time series, $\mathrm{S}$ is the standard deviation of time series, $\mathrm{H}$ is hurst Index, A is a constant. On the basis of the following formula takes the number determined by the least squares method to obtain $\mathrm{H}$ Hurst index.

\section{SUMMARY}

Tracking of moving target is a key issue in computer vision research. It has very important practical value and broad development prospects in the field of military visual guidance, robot vision navigation, industrial product testing, medical diagnosis, traffic monitoring, virtual reality and so on. The difficulty of target tracking is the complexity of the object movement in the real environment, the complexity of video data, and the reliable tracking results in complex environment. The design of a robust, realtime, robust tracking algorithm has been a long-term goal in the field of research.

The main results of this paper are:

(1)Based on already captured video files, image segmentation is realized by using MATLAB programming;

(2)Out of image segmentation using background subtraction method to obtain a foreground image moving target;

(3)The prospect of an image obtained by median filtering, gray-scale processing and edge detection to give contour moving target;
(4)Computing equivalent moving target contour area and perimeter equivalent, and then obtain the equivalent diameter;

(5)By detecting and analyzing parameters such as equivalent diameter coefficient of variation;

(6)The meaning and difference between flexible motion and rigid motion are understood, through the analysis of the coefficient of variation, to get the moving target is a flexible or rigid movement.

\section{REFERENCES}

Camille Vidal,Bruno Jedynak. Learning to Match: Deriving Optimal Template-Matching Algorithms from Probabilistic Image Models [J]. International Journal of Computer Vision. 2010 (2)

Chen Y,Yu SS,Fan J, et al.An improved Color-Based Particle Filter for Object Tracking. The 2nd International Conference on Genetic and Evolutionary Computing. 2008

D. Roller, K. Daniilidis,H. H. Nagel. Model-based object tracking in monocular image sequences of road traffic scenes [J]. International Journal of Computer Vision. 1993 (3)

Li CM,Xu CY,Gui CF,et al.Level set evolution without reinitialization: A new variational formulation. IEEE Computer Society Conference on Computer Vision and Pattern Recognition. 2005

Matwyschuk A, Ambs P, Christnacher F.Analysis of an optical target tracking system implementing a snake-based segmentation. Proceedings of the Society of Photo-optical Instrumentation Engineers. 2003

Osher S,Sethian JA.Fronts Propagating with curvatureDependent Speed. Journal of Computational Physics. 1988

Sen-Ching S Cheung,Chandrika Kamsth.Robust techniques for background subtraction in urban traffic video. Proceedings of Electronic Imaging:Visual Communications and Image Processing . 2004

Tyrsa VY, Sergiyenko OY,Tyrsa VV, et al.Mobile robot navigation by later scanning means. The 3rd International Conference on Cybernetics and Information Technologies, Systems and Applications/4th International Conference on Computing, Communications and Control Technologies. 2006

Tsai A,Yezzi JA,Willsky AS.Curve evolution implementation of the Mumford-Shah functional for image segmentation, denoising, interpolation, and magnification. IEEE Transactions on Image Processing. 2001

Vicent Caselles, Francine Catte, Tomen Coll, et al. A geometric model for active contours in image processing. Numerische Mathematik. 1993 